\title{
Novel VANGL1 Gene Mutations in 144 Slovakian, Romanian and German Patients with Neural Tube Defects
}

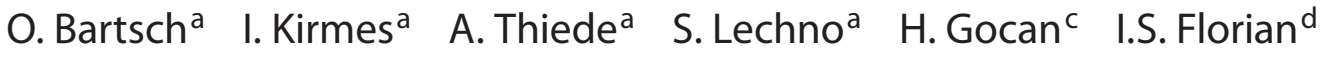 \\ T. Haaf ${ }^{b} \quad$ U. Zechner ${ }^{a} \quad$ L. Sabova ${ }^{\text {e }} \quad$ F. Horn ${ }^{f}$
}

a Institute of Human Genetics, University Medical Center of the Johannes Gutenberg University Mainz, Mainz, and ${ }^{b}$ Institute of Human Genetics, Julius Maximilians University, Würzburg, Germany; ${ }^{\mathrm{C}}$ Department of Pediatric Surgery, and d Section of Neurosurgery, University of Cluj-Napoca, Cluj-Napoca, Romania; ${ }^{\mathrm{e}}$ 2nd Department of Pediatrics, and fDepartment of Pediatric Surgery, Comenius University, Bratislava, Slovakia

\section{Key Words}

Neural tube defects • Planar cell polarity pathway •

VANGL1 $\cdot$ Mutational hot spots

\begin{abstract}
Neural tube defects (NTDs) are a group of congenital malformations of the central nervous system occurring at an average rate of 1 per 1,000 human pregnancies worldwide. Numerous genetic and environmental factors are discussed to be relevant in their etiology. In mice, mutants in $>200$ genes including the planar cell polarity (PCP) pathway are known to cause NTDs, and recently, heterozygous mutations in the human VANGL1 gene have been described in a small subset of patients with NTDs. We performed a VANGL1 mutation analysis in 144 unrelated individuals with NTDs from Slovakia, Romania and Germany and identified 3 heterozygous missense mutations: c.613G >A (p.Gly205Arg) with an open spina bifida (lumbosacral meningomyelocele), c.557G $>\mathrm{A}$ (p.Arg186His) with a closed spina bifida (tethered cord and spinal lipoma) and c.518G $>$ A (p.Arg173His) with an unknown NTD. The c.613G $>$ A mutation was also found in a healthy sibling. None of the mutations were described previously. Findings support that heterozygous VANGL1 mutations represent hypomorphs or conditional mutants predisposing to NTDs and occur at a frequency of approximately $2.1 \%$ of
\end{abstract}

open and closed spinal NTDs. The mutations (p.Arg173His, p.Arg186His, p.Gly205Arg) modified conserved regions of the VANGL1 protein and shared similarities with previously described mutants, providing further evidence for the presence of mutational hot spots in these patients.

Copyright $\odot 2012$ S. Karger AG, Basel

Human neural tube defects (NTDs) are among the most common and serious birth defects with an incidence averaging 1 per 1,000 pregnancies worldwide [Copp et al., 2003; Bassuk and Kibar, 2009]. NTDs affect the central nervous system, including the spinal cord, skull and brain and may also involve the linings of the brain, the vertebral branches, muscle, and skin. The phenotypic spectrum includes the severe 'open' forms of spina bifida and the milder 'closed' forms (also called dysraphic conditions). NTDs result from defective neurulation. Neurulation is conventionally divided into a primary and a secondary phase: in primary neurulation, the neural plate is formed by shaping, folding and midline fusion into the neural tube, which then is covered by ectoderm [Copp et al., 2003]. Primary neurulation creates the brain and nearly all of the human spinal cord, down to the upper sacral level. Defective primary neurulation typically leads to open forms of NTDs: cranior-

\section{KARGER}

Fax +4161306 1234

E-Mail karger@karger.ch

www.karger.com (c) 2012 S. Karger AG, Basel

$1661-8769 / 12 / 0032-0076 \$ 38.00 / 0$

Accessible online at:

www.karger.com/msy
Prof. Dr. Oliver Bartsch

Institute of Human Genetics, University Medical Center Mainz

Langenbeckstrasse 1

DE-55101 Mainz (Germany)

Tel. +49 613117 5791, E-Mail oliver.bartsch@unimedizin-mainz.de 
Table 1. Missense mutations altering highly conserved amino acid residues of VANGL1 and identified in patients with NTDs in this and previous studies

\begin{tabular}{llll}
\hline Position & Mutation & Effect & Reference \\
\hline Exon 3 & c.248C $>\mathrm{T}$ & p.Ser83Leu & Kibar et al., 2009 \\
Exon 3 & c.485T $>$ C & p.Phe153Ser & Kibar et al., 2009 \\
Exon 3 & c.518G $>$ A & p.Arg173His & this study \\
Exon 3 & c.542G $>$ A & p.Arg181Gln & Kibar et al., 2009 \\
Exon 3 & c.557G $>$ A & p.Arg186His & this study \\
Exon 3 & c.604C $>$ T & p.Leu202Phe & Kibar et al., 2009 \\
Exon 3 & c.613G $>$ A & p.Gly205Arg & this study \\
Exon 3 & c.715G $>$ A & p.Val239Ile & Kibar et al., 2007 \\
Exon 4 & c.821G $>$ A & p.Arg274Gln & Kibar et al., 2007 \\
Exon 5 & c.984T $>$ C & p.Met328Thr & Kibar et al., 2007 \\
Exon 6 & c.1210G $>$ T & p.Ala404Ser & Kibar et al., 2009 \\
\hline
\end{tabular}

Mutations were absent in controls. It is thought that these mutations do not cause NTDs on their own, but do so in oligogenic or multifactorial combinations.

rhachischisis (the most severe failure of closure already at the hindbrain/cervical boundary, 'closure 1 defect'), anencephaly (the failure of closure at the most anterior neuropore, termed 'closure 3 defect') and spina bifida (the failure of closure at the posterior neuropore) [Botto et al., 1999; Copp et al., 2003]. Secondary neurulation is defined as the formation of the caudal portion of the neural tube from the tail bud (also called caudal eminence) and occurs without folding. Secondary neurulation creates most of the sacral and all of the coccygeal tissues of the spinal cord [Copp et al., 2003]. Defective secondary neurulation results in closed forms of spina bifida, where the emerging spinal cord tissue fails to be separated from the other tissues of the tail bud. A 'tethered' spinal cord is indicative of a closed spina bifida and always affects the lower part of the spine.

The etiology of NTDs is multifactorial. Numerous factors contributing to embryonal neural tube closure have been identified, including nutritional factors (folic acid, inositol) and genes in different signaling systems, in particular, the planar cell polarity (PCP) pathway [Copp et al., 2003; Simons and Mlodzik, 2008]. Mutants in more than 200 genes cause NTDs in mice [Harris and Juriloff, 2010]. In addition to null mutants, many hypomorphs or conditional mutants in the PCP pathway have been identified, and some mutations do not cause NTDs on their own, but do so in digenic, trigenic and oligogenic combinations [Torban et al., 2008; Harris and Juriloff, 2010]. It is thought that human NTDs arise in a similar fashion. Genes in the
PCP pathway include Frizzled, Scrb1, Dishevelled, Celsr1/ Flamingo, Prickle, Diego and Strabismus/VanGogh (Stbm/ Vang) [Copp et al., 2003; Harris and Juriloff, 2010]. In the Looptail mouse, homozygotes for some Vangl2 mutants (Ser464Asn, Asp255Glu) display a lethal NTD, craniorrhachischisis [Kibar et al., 2001; Iliescu et al., 2011], whereas homozygotes for a hypomorphic Vangl2 allele (Arg259Leu) may also show milder, spinal NTDs [Guyot et al., 2011]. The first human mutations in developmental genes implicated in the causation of NTDs were detected in the VANGL1 gene [Kibar et al., 2007]. So far 8 heterozygous VANGL1 mutations (table 1) have been reported in 817 patients with NTDs (1\%, 8/817) [Kibar et al., 2007, 2009].

We performed a study of the human VANGL1 gene in 144 unrelated Slovakian, Romanian and German patients and identified 3 novel heterozygous missense mutations (p.Arg173His, p.Arg186His, and p.Gly205Arg). The mutations shared similarities with previously described mutants, suggesting the presence of mutational hot spots. Findings provide additional evidence that VANGL1 missense mutations predispose to open and closed spinal NTDs.

\section{Materials and Methods}

\section{Patients}

The VANGL1 mutation analyses were performed as a clinical service with board approved use of the data for research. For all controls, written consent had been obtained for the use of surplus DNA in medical research. We studied 144 unrelated individuals (76 females, 68 males), including 51 probands from the University Medical Center, Mainz, Germany; 84 patients from the Departments of Pediatrics and Pediatric Surgery, Comenius University, Bratislava, Slovakia; and 9 patients receiving spina bifida surgery in Cluj-Napoca, Romania. The Mainz samples included muscle fiber DNA from 2 abortions with NTDs and amniotic fluid DNA from 2 fetuses. In most cases, DNA from unaffected relatives was not available. For 1 mutation-positive Slovakian patient, we could obtain lymphocyte DNAs from his healthy mother and sister, but detailed clinical assessment was not performed, and therefore, minimal phenotypic anomalies cannot be excluded. Patients were born between 1972 and 2009 (85\%, 1991 and later) and recruited between 2004 and 2009. All NTDs were clinically relevant requiring surgery. Detailed clinical information was only available for a subset of patients, including open and closed NTDs: anencephaly, encephalocele, meningomyelocele, tethered cord, spinal lipoma, and sinus dermalis. The folic acid status of the mothers is known for the Slovakian and Romanian patients. In all these cases, periconceptional folic acid supplementation was either not given or given too late (after the pregnancy had been diagnosed). The control group consisted of 357 individuals (169 females, 188 males) born between 1948 and 2008 (93\%, 1991 and later) and collected between 2003 and 2010 at the Institute of Human Genetics, Mainz, Germany. None of the controls suffered from a NTD. 
Fig. 1. Mutations in patients with NTDs. A Electropherograms showing 3 novel VANGL1 mutations: c.518G $>$ A (predicting p.R173H), c.557G $>$ A (p.R186H), and c.613G $>$ A (p.G205R). Arrows indicate base substitutions. Note the heterozygous state of the mutations. B Locations of all relevant VANGL1 sequence variants identified in patients with NTDs in this and previous studies [Kibar et al., 2007, 2009]. Eleven unequivocal mutations altering conserved amino acid residues are shown in black. Exons (but not introns and UTRs) are drawn to scale. Note the mutational hot spot in exon 3.

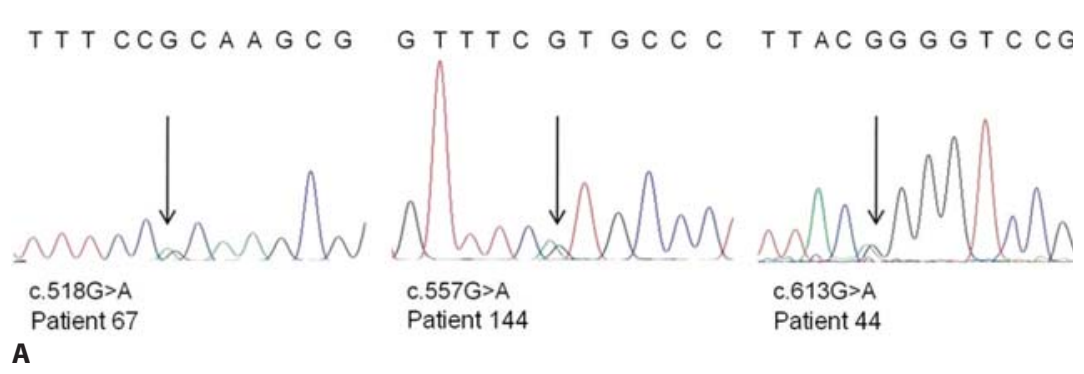

A

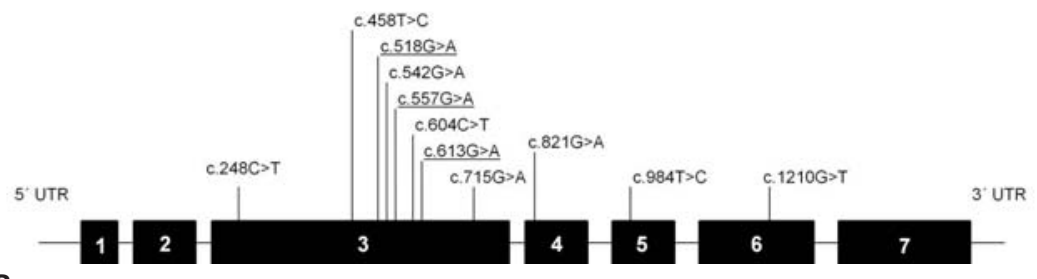

B

\section{Genetic Analysis}

The human VANGL1 NCBI reference sequence has accession number NM_138959.2, and the number of amino acids in the protein is 524 . DNA was extracted from peripheral blood by standard high-salt precipitation method. Primers for amplification of the 7 coding exons, including at least 20 bases of surrounding intronic sequence, were designed using the Primer3 program (http:// frodo.wi.mit.edu/primer3/). Primer sequences and PCR conditions are available upon request. PCR products were purified using the ExoSap method and tagged with M13 universal primers. Direct bi-directional sequencing of all coding exons was performed with an automated capillary sequencer (Beckman Coulter CEQ $^{\text {TM }} 8000$ Genetic Analysis System). Electropherograms (fig. 1A) were examined visually and using Mutation Surveyor, version 3.2 (SoftGenetics, State College, Pa., USA). Potential pathogenic effects of the mutations were predicted using the classification tools MutationTaster (http://www.mutationtaster. org) [Schwarz et al., 2009] and PolyPhen (http://genetics.bwh. harvard.edu/pph/) with UniProt accession number Q8TAA9 for VANGL1. For all mutations, conservation across species was determined (fig. 2B). In the controls, we studied only exon 3, which harbored all mutations in this study.

\section{Results}

Genetic analysis of VANGL1 identified 3 heterozygous missense mutations (c.518G $>$ A, c. $557 \mathrm{G}>\mathrm{A}$ and c.613G $>\mathrm{A}$ ) that were not listed in SNP databases (http://www. ensembl.org/, www.ncbi.nlm.nih.gov/projects/SNP/) and absent in 357 controls (fig. 1, table 1). The mutations had not been reported previously. Based on their location in the protein and their alteration of perfectly or highly con- served amino acid residues (fig. 2), all 3 sequence changes were predicted by conventional programs as probably disease causing. The 3 patients with mutations had uninformative family histories.

The c.518G $>$ A (p.Arg173His) mutation was detected in a Slovakian male born in 1987 with a sporadic NTD; other clinical data are not available. The arginine residue at position 173 is located in the intracellular loop between transmembrane domains 2 and 3 (TM2 and TM3) (fig. 2A) and showed perfect conservation in all analyzed species including C. elegans (fig. 2B). The exchange of arginine to histidine is conservative, as both amino acids are hydrophilic and basic, but Arginine is strongly charged (pKa 12.5) and has a small aliphatic side chain, whereas histidine has a near neutral pKa (7.6) and contains a large aromatic side chain with an imidazole ring. MutationTaster and PolyPhen, respectively, predicted p.Arg173His as probably disease causing $(\mathrm{p}=0.9999)$ and damaging (PSIC score difference 2.295).

The c.557G $>$ A (p.Arg186His) mutation was present in a Slovakian female born in 2003 with sporadic spinal lipoma and tethered cord. This exchange of arginine to histidine is located in transmembrane domain 3 next to the intracellular loop between TM2 and TM3 (fig. 2A). The modified residue showed perfect conservation in all analyzed species (fig. 2B). The p.Arg186His mutant was rated by MutationTaster as probably disease causing ( $\mathrm{p}=$ 0.9999) and by PolyPhen as possibly damaging due to an improper substitution in the transmembrane region. 
Fig. 2. A Topological model of the VANGL1 protein (length, 524 amino acids) in the cell membrane. Mutants identified in this and previous studies are indicated in black. Mutants in this study are framed. Vangl2 mutations described in Looptail ( $L p)$ mice are depicted in grey. Note the clustering of mutations at the intra- and extracellular borders of the transmembrane domains. B Partial alignment of hVANGL1 and 11 orthologs $(\mathrm{h}=$ Human, $c=$ chimp, $r=$ rat, $m=$ mouse, $\mathrm{ra}=$ rabbit, $\mathrm{d}=\mathrm{dog}, \mathrm{ca}=\mathrm{cat}, \mathrm{co}=\mathrm{cow}$, $\mathrm{ch}=$ chicken, $\mathrm{T}=$ tetraodon/pufferfish, $\mathrm{d}=$ Drosophila, $\mathrm{c}=C$. elegans). Note the conserved and altered amino acids. Conserved residues are highlighted. Figure adapted from Kibar et al. [2009].

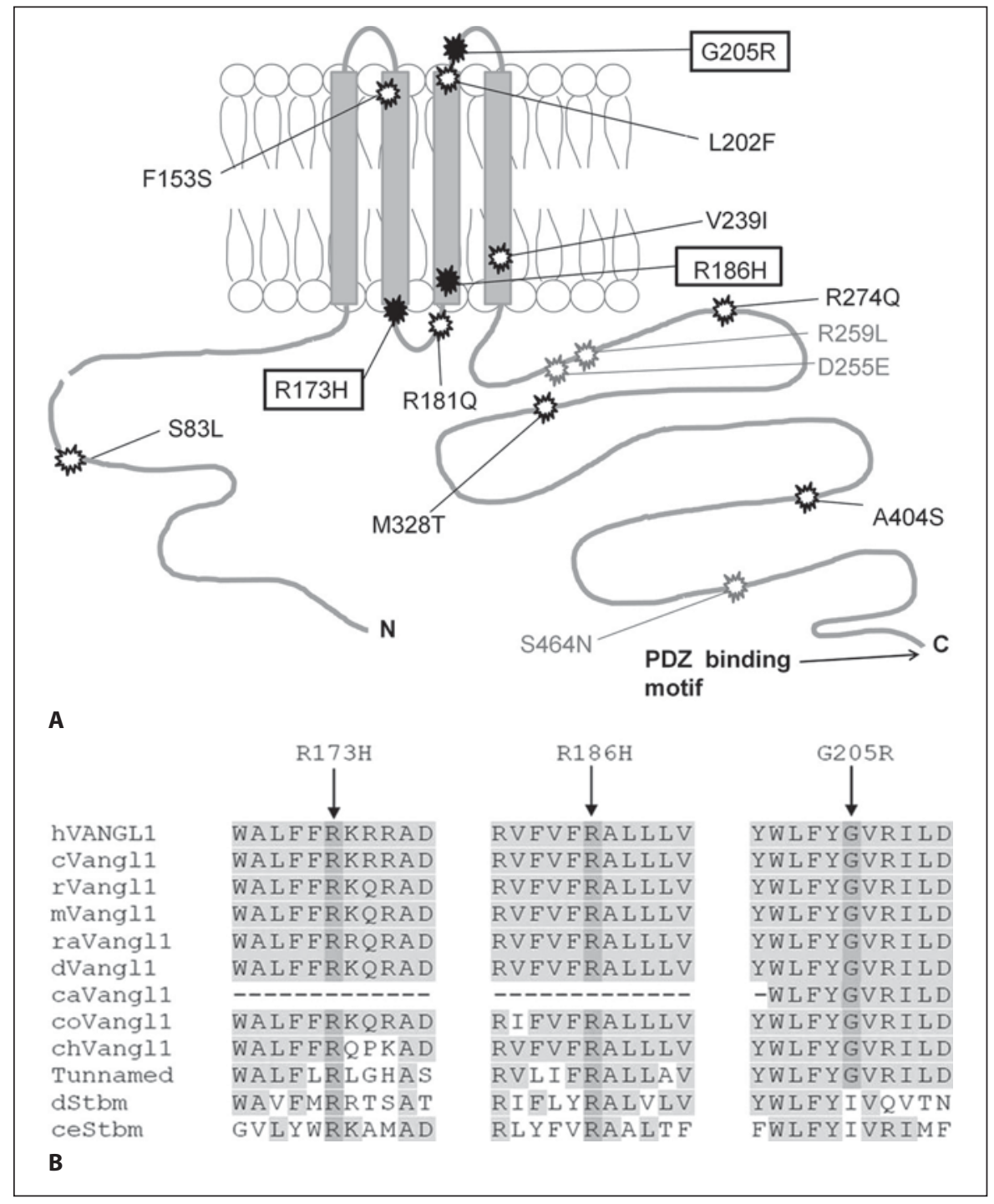

The c.613G $>$ A (p.Gly205Arg) mutation was identified in a Slovakian male born in 1991 with a sporadic lumbosacral meningomyelocele. It was also present in the patient's healthy younger sister and absent in his mother. The father was not available for study. Hence, the mutation was probably paternally inherited, but maternal germ line mosaicism cannot be excluded. The glycine residue at position 205 resides in the extracellular loop between TM3 and TM4 and is perfectly conserved among vertebrates (fig. 2A, B). The Gly-to-Arg substitution is nonconservative. Glycine is hydrophobic and neutrally charged, whereas arginine is hydrophilic and positively charged. MutationTaster and PolyPhen, respectively, evaluated p.Gly205Arg as probably disease causing ( $\mathrm{p}=$ 0.9998) and damaging (PSIC score difference 2.082).
The known VANGL1 missense variant c.523C $>$ T (p.Arg175Trp) was identified in a healthy control. The p.Arg175Trp variant was previously described as a rare mutation present in both NTD patients $(n=3)$ and controls $(\mathrm{n}=1)$ [Kibar et al., 2009]. Using mutation classification tools MutationTaster and PolyPhen, respectively, p.Arg175Trp was predicted to be a polymorphism ( $\mathrm{p}=$ 0.9884 ) and benign (PSIC score difference 0.492). Two additional novel heterozygous variants were considered as likely benign: 2 patients carried the noncoding variant c.1314 $+12 \mathrm{C}>\mathrm{T}$ and 1 patient displayed the silent variant c.933C $>$ A (p.Val311Val); these variants have not yet been described (table 2). Further, we observed 6 SNPs (rs10754330, rs10923176, rs10923177, rs12739928, rs41275546, and rs4839469). 
Table 2. Rare VANGL1 missense variants of unknown significance (UVs) and silent variants not reported recently

\begin{tabular}{|c|c|c|c|c|c|c|}
\hline \multirow[t]{2}{*}{ Position } & \multirow[t]{2}{*}{ Variant } & \multirow[t]{2}{*}{ Effect } & \multicolumn{2}{|c|}{ NTD patients } & \multicolumn{2}{|l|}{ Controls } \\
\hline & & & $\begin{array}{l}\text { this study } \\
(\mathrm{n}=144)\end{array}$ & $\begin{array}{l}\text { Kibar et al. } \\
{[2009](n=673)}\end{array}$ & $\begin{array}{l}\text { this study } \\
(\mathrm{n}=357)\end{array}$ & Kibar et al. [2009] \\
\hline \multicolumn{7}{|c|}{ Rare UVs, some could possibly represent mild mutations } \\
\hline Exon 2 & c. $73 \mathrm{G}>\mathrm{A}$ & p.Glu25Lys & 0 & 1 & 0 & $0(n=1,346)$ \\
\hline Exon 3 & c. $523 \mathrm{C}>\mathrm{T}$ & p.Arg175Trp & 0 & 3 & 1 & $1(\mathrm{n}=355)$ \\
\hline Exon 4 & c. $868 \mathrm{~T}>\mathrm{C}$ & p.Tyr290His & 0 & 1 & 0 & $0(\mathrm{n}=1,355)$ \\
\hline Exon 7 & c. $1401 \mathrm{~T}>\mathrm{G}$ & p.Asp468Glu & 0 & 1 & 0 & $0(\mathrm{n}=1,255)$ \\
\hline \multicolumn{7}{|c|}{ Novel silent variants, likely benign } \\
\hline Exon 4 & c. $933 \mathrm{C}>\mathrm{A}$ & synonymous & 1 & & not studied & \\
\hline IVS6 & c. $1314+12 \mathrm{C}>\mathrm{T}$ & intronic & 2 & & not studied & \\
\hline
\end{tabular}

UVs changed poorly conserved amino acid residues but could possibly represent mild mutations. Silent variations were synonymous or intronic and likely benign.

\section{Discussion}

In this cohort of 144 patients, we identified 3 heterozygous missense mutations in VANGL1 (p.Arg173His, p.Arg186His, p.Gly205Arg) that were absent in controls. Each mutation modified evolutionary conserved amino acid residues in the membrane area of the VANGL1 protein and predicted functional consequences. We consider most likely that these are pathogenic mutations that may display a mutant phenotype under certain conditions (in a multifactorial setting).

In previous studies of patients with NTDs, 8 likely pathogenic VANGL1 mutations modifying conserved amino acid residues (fig. $1 \mathrm{~B}$ and $2 \mathrm{~A}$, table 1 ) and 5 unclassified rare variants altering poorly conserved residues of VANGL1 (table 2) were identified [Kibar et al., $2007,2009]$. The frequency of $2.1 \%$ (3/144) VANGL1 mutations in this cohort is in the same magnitude as the detection rate of $0.9 \%(8 / 817)$ in previous studies [Kibar et al., 2007, 2009].

By impact on protein sequence, no VANGL1 frameshift or nonsense mutations were identified. All 11 disease-associated variants in this and previous studies were heterozygous missense mutations. Eight mutations (73\%) were located in exon 3, and of these, 7 altered conserved residues in the membrane-associated part of the protein, suggesting a common pathomechanism of these mutations and indicating a mutational hot spot associated with NTDs. The mutants p.Arg173His, p.Arg186His and the previously reported p.Arg181Gln [Kibar et al., 2009] cluster at the intracellular loop connecting the TM2 and TM3, and they all modify arginine residues conserved in all analyzed species, including nematodes (fig. 2). Similarly, the p.Gly205Arg mutant resides in a TM domain/ loop boundary region, only 3 amino acids away from the previously described p.Leu202Phe mutation [Kibar et al., 2009]. The functional effects of these mutations are still unknown, but they could reduce the membrane targeting of the VANGL1 protein. Another mutation mapping to a TM domain/loop boundary region (p.Val239Ile) was already shown to be a complete loss-of-function mutant using vangl2/tri-MO knockdown/rescue experiments in zebrafish [Reynolds et al., 2010]. Furthermore, 3 (p.Arg135Trp, p.Arg177His, p.Val178Ile) of 7 mutations in the human VANGL2 gene in patients with NTDs resided in TM domain/loop boundary regions [Kibar et al., 2011]. Another possible mutational hot spot region in VANGL proteins may map downstream of the TM4 domain at the beginning of the C-terminal cytoplasmic tail. The VANGL1(Arg274Gln) mutant [Kibar et al., 2007], 3 VANGL2 mutants (p.Leu242Val, p.Thr247Met, p.Arg270His) [Kibar et al., 2011] and 2 of the 3 murine Vangl2 mutations (Asp255Glu, Arg259Leu) [Iliescu et al., 2011; Guyot et al., 2011] locate to this region and 3 of these 6 mutations modify arginine residues (fig. 2A). Functional characterization of the Looptail mouse mutant Arg259Leu by expression in polarized Madin-Darby canine kidney cells resulted in a severe loss of VANGL2 function by defective targeting of the protein to the cell membrane, concurrent with retention of the mutant pro- 
tein in the endoplasmic reticulum, and in a significantly reduced protein half-life [Iliescu et al., 2011]. Biochemical studies of the Looptail mutants Asp255Glu and Arg259Leu have also suggested a role of this other mutational hot spot region in protein folding and membrane targeting [Gravel et al., 2010]. Hence, the pathogenicity of the mutations in this study (p.Arg173His, p.Arg186His, p.Gly205Arg) is supported by (i) their absence in controls, (ii) the in silico findings using mutation classification tools, (iii) the modification of arginine residues in 2 cases, and (iv) by their localization to the area of a functionally characterized loss-of-function mouse mutant, Arg259Leu.

The mutations in this cohort all occurred with sporadic nonsyndromic NTDs: p.Gly205Arg with an open spinal NTD (lumbosacral meningomyelocele), p.Arg186His with a closed spinal NTD (tethered cord and spinal lipoma) and p.Arg173His with an unknown NTD. The sporadic occurrence could be due to chance because familial NTDs are rare and the recurrence risk in 1st degree relatives is only 3-4\% [Record and McKeown, 1950]. However, the p.Gly205Arg mutation was also identified in the healthy sister of the patient with the meningomyelocele, and there have been previous reports of VANGL1 mutations in healthy relatives [Kibar et al., 2007, 2009]. This suggests that heterozygous VANGL1 mutations represent hypomorphs or conditional mutants predisposing to NTDs, not fully penetrant mutations. Additional stud- ies on the functional effects of the mutant proteins are needed.

Further studies are also needed to determine the significance of rare missense variants changing poorly conserved amino acid residues of VANGL1 (table 2). We identified one healthy control (1/357) with a variant of the poorly conserved residue 175 of VANGL1, p.Arg175Trp. This variant had been previously identified both in patients with NTDs (3/673) and a control (1/355) and had been classified as likely normal [Kibar et al., 2009]. However, the proximity of p.Arg175Trp to other VANGL1 mutants modifying arginines (p.Arg173His, p.Arg181Gln, p.Arg186His) is suggestive that this could be another hypomorphic allele for NTDs.

\section{Acknowledgements}

The authors thank the patients and controls for their consent to this study. We also wish to thank Konstanze Gebauer (Institute of Human Genetics, University Medical Center, Mainz, Germany) for primer design, Cornelia Poarangan and Farah Izadpanah (Institute of Human Genetics, University Medical Center, Mainz, Germany) for excellent technical assistance, and Dr. Vasilica Plaiasu (University of Medicine and Pharmacy Carol Davila, Bucharest, Romania), Paula Percsi (University of Cluj, Romania) and Dr. Georg Kutschke (Department of Pediatrics, University Medical Center, Mainz, Germany) for contributing materials for the present study.

\section{References}

Bassuk A, Kibar A: Genetic basis of neural tube Iliescu A, Gravel M, C Horth, Kibar Z, Gros P: defects. Semin Pediatr Neurol 16:101-110 (2009).

Botto L, Moore C, Khour M, Erickson J: Neuraltube defects. N Engl J Med 341:1509-1519 (1999).

Copp A, Greene N, Murdoch J: The genetic basis of mammalian neurulation. Nat Rev Genet 4:784-793 (2003).

-Gravel M, Iliescu A, Horth C, Apuzzo S, Gros P: Molecular and cellular mechanisms underlying neural tube defects in the loop-tail mutant mouse. Biochemistry 49:3445-3455 (2010).

- Guyot M, Bosoi C, Kharfallah F, Reynolds A, Drapeau P, et al: A novel hypomorphic Looptail allele at the planar cell polarity Vangl2 gene. Dev Dyn 240:839-849 (2011).

-Harris MJ, Juriloff DM: An update to the list of mouse mutants with neural tube closure defects and advances toward a complete genetic perspective of neural tube closure. Birth Defects Res A Clin Mol Teratol 88:653-669 (2010). Loss of membrane targeting of Vangl proteins causes neural tube defects. Biochemistry 50:795-804 (2011).

Kibar Z, Vogan K, Groulx N, Justice M, Underhill D, Gros P: Ltap, a mammalian homolog of Drosophila Strabismus/Van Gogh, is altered in the mouse neural tube defect mutant Loop-tail. Nat Genet 28:251-255 (2001).

Kibar Z, Torban E, McDearmid J, Reynolds A, Berghout J, et al: Mutations in VANGL1 are associated with neural tube defects in humans. New Engl J Med 356:1432-1437 (2007).

Kibar Z, Bosoi C, Kooistra M, Salem S, Finnell R, et al: Novel mutations in VANGL1 in neural tube defects. Hum Mutat 30:706-715 (2009).

- Kibar Z, Salem S, Bosoi C, Pauwels E, De Marco P, et al: Contribution of VANGL2 mutations to isolated neural tube defects. Clin Genet 80:76-82 (2011)
Record RG, McKeown T: Congenital malformations of the central nervous system. III. Risk of malformation in sibs of malformed individuals. Br J Soc Med 4:217-220 (1950).

Reynolds A, McDearmid J, Lachance S, De Marco P, Merello E, et al: VANGL1 rare variants associated with neural tube defects affect convergent extension in zebrafish. Mech Dev 127:385-392 (2010).

Schwarz J, Rödelsperger C, Schuelke M, Seelow D: MutationTaster evaluates disease-causing potential of sequence alterations. Nat Methods 7:575-576 (2010).

- Simons M, Mlodzik M: Planar cell polarity signaling: from fly development to human disease. Annu Rev Genet 42:517-540 (2008).

- Torban E, Patenaude A, Leclerc S, Rakowiecki S, Gauthier S, et al: Genetic interaction between members of the Vangl family causes neural tube defects in mice. Proc Natl Acad Sci USA 105:3449-3454 (2008). 